\title{
Study protocol: multi-parametric magnetic resonance imaging for therapeutic response prediction in rectal cancer
}

Trang Thanh Pham ${ }^{1,2,3,4,7^{*}}$, Gary Liney ${ }^{1,3,4,9}$, Karen Wong ${ }^{1,3,4}$, Robba Rai ${ }^{1,4}$, Mark Lee ${ }^{1,3}$, Daniel Moses ${ }^{3,5}$, Christopher Henderson ${ }^{3,6,7}$, Michael Lin ${ }^{3,7,8}$, Joo-Shik Shin ${ }^{6,7}$ and Michael Bernard Barton 1,3,4

\begin{abstract}
Background: Response to neoadjuvant chemoradiotherapy (CRT) of rectal cancer is variable. Accurate imaging for prediction and early assessment of response would enable appropriate stratification of management to reduce treatment morbidity and improve therapeutic outcomes. Use of either diffusion weighted imaging (DWI) or dynamic contrast enhanced (DCE) imaging alone currently lacks sufficient sensitivity and specificity for clinical use to guide individualized treatment in rectal cancer. Multi-parametric MRI and analysis combining DWI and DCE may have potential to improve the accuracy of therapeutic response prediction and assessment.

Methods: This protocol describes a prospective non-interventional single-arm clinical study. Patients with locally advanced rectal cancer undergoing preoperative CRT will prospectively undergo multi-parametric MRI pre-CRT, week 3 CRT, and post-CRT. The protocol consists of DWI using a read-out segmented sequence (RESOLVE), and DCE with pre-contrast T1-weighted (VIBE) scans for T1 calculation, followed by 60 phases at high temporal resolution (TVIST) after gadoversetamide injection. A 3-dimensional voxel-by-voxel technique will be used to produce colour-coded ADC and $K^{\text {trans }}$ histograms, and data evaluated in combination using scatter plots. MRI parameters will be correlated with surgical histopathology. Histopathology analysis will be standardized, with chemoradiotherapy response defined according to AJCC 7th Edition Tumour Regression Grade (TRG) criteria. Good response will be defined as TRG 0-1, and poor response will be defined as TRG 2-3.
\end{abstract}

Discussion: The combination of DWI and DCE can provide information on physiological tumour factors such as cellularity and perfusion that may affect radiotherapy response. If validated, multi-parametric MRI combining DWI and DCE can be used to stratify management in rectal cancer patients. Accurate imaging prediction of patients with a complete response to CRT would enable a 'watch and wait' approach, avoiding surgical morbidity in these patients. Consistent and reliable quantitation from standardised protocols is essential in order to establish optimal thresholds of ADC and $K^{\text {trans }}$ and permit the role of multi-parametric MRI for early treatment prediction to be properly evaluated.

Trial registration: Australian New Zealand Clinical Trials Registry (ANZCTR) number ACTRN12616001690448 (retrospectively registered 8/12/2016).

Keywords: MRI, Rectal cancer, Radiotherapy, Response, Chemoradiotherapy, Diffusion weighted imaging, Dynamic contrast enhanced, Diffusion, Perfusion

\footnotetext{
* Correspondence: Trang.Pham@health.nsw.gov.au

'Department of Radiation Oncology, Liverpool Cancer Therapy Centre, Liverpool Hospital, South Western Sydney Local Health District, Sydney, Australia

${ }^{2}$ Sydney West Radiation Oncology Network, Westmead, Blacktown and Nepean Hospitals, Sydney, Australia

Full list of author information is available at the end of the article
}

\section{Ciömed Central}

(c) The Author(s). 2017 Open Access This article is distributed under the terms of the Creative Commons Attribution 4.0 International License (http://creativecommons.org/licenses/by/4.0/), which permits unrestricted use, distribution, and reproduction in any medium, provided you give appropriate credit to the original author(s) and the source, provide a link to the Creative Commons license, and indicate if changes were made. The Creative Commons Public Domain Dedication waiver (http://creativecommons.org/publicdomain/zero/1.0/) applies to the data made available in this article, unless otherwise stated. 


\section{Background}

Locally advanced rectal cancer (LARC) requires multimodality treatment consisting of neoadjuvant chemoradiotherapy (CRT) and standardized surgical technique (total mesorectal excision) [1-3]. Response to neoadjuvant therapy is variable; $15-27 \%$ of patients will have a pathologic complete response (pCR) [4], whilst 25-45\% will have a poor response with minimal tumour regression [5]. In patients with locally advanced rectal cancer undergoing CRT and surgery, $45 \%$ of patients will require permanent colostomy [6]. Accurate imaging for prediction and early assessment of response would enable appropriate stratification of management to reduce treatment morbidity and improve therapeutic outcomes. In patients with a clinical complete response to CRT, substitution of surgery by a 'watch-and-wait' approach has emerged as a management option [7-9]. Prediction of poor response could permit trials of dose escalation strategies or curtailment of futile treatment.

Functional magnetic resonance imaging (MRI) has shown promising results for prediction of CRT response in rectal cancer. Diffusion weighted imaging (DWI) has demonstrated greater potential compared with morphologic T2-weighted (T2-w) imaging for the assessment of therapeutic response in rectal cancer patients [10]. However, a systematic review by Joye et al. found that pre-CRT quantitative DWI alone was unable to predict PCR with sensitivity and specificity of $69 \%$ and $68 \%$, respectively. Quantitative DWI post-CRT had sensitivity and specificity of $78-80 \%$ and $72-78 \%$, respectively, for detecting pCR [11]. Some dynamic contrast enhanced (DCE) MRI studies have shown that higher contrast exchange rate pretreatment, as indicated by higher $\mathrm{K}^{\text {trans }}$, is associated with better response to CRT $[12,13]$. One study did not find a correlation between pre-treatment $\mathrm{K}^{\text {trans }}$ and therapeutic response [14]. Use of either DWI or DCE alone currently lacks sufficient accuracy for clinical use to guide individualized treatment in rectal cancer.

Multi-parametric MRI combining DWI and DCE may have potential to improve the accuracy of therapeutic response prediction and assessment. Most published studies describe mean values of a region of interest (ROI) from either DWI or DCE. Single parameter measurements, such as mean apparent diffusion co-efficient (ADC) or $\mathrm{K}^{\text {trans }}$, do not adequately reflect intra-tumour heterogeneity. A three-dimensional analysis of the tumour volume would provide information on tumour heterogeneity. Development and standardization of multi-parametric imaging protocols is required to provide robust serial imaging datasets and reliable quantitative assessment of treatment response.

\section{Study hypothesis}

Multi-parametric MRI, consisting of DWI and DCE, performed pre-, during- and post- neoadjuvant CRT is predictive of treatment outcome in locally advanced rectal cancer, with histopathology being the standard reference.

\section{Methods/design \\ Study objectives \\ Primary objective}

To prospectively evaluate pre-, during- and post-CRT multi-parametric MRI (DWI and DCE) at 3 Tesla for therapeutic response prediction in LARC. MRI biomarkers will be correlated with histopathology tumour regression grade (TRG).

\section{Secondary objectives}

1. To prospectively evaluate the role of DCE MRI for therapeutic response prediction and assessment in LARC.

2. To prospectively evaluate the role of DWI MRI (RESOLVE) for therapeutic response prediction and assessment in LARC.

3. To evaluate the different contributions of MRI and PET for therapeutic prediction and assessment in LARC.

4. To correlate MRI biomarkers with 2 year disease-free survival and overall survival.

\section{Study design}

The study design is a prospective, single-arm, cohort study to investigate the value of multi-parametric MRI (combining DWI and DCE) in the prediction and assessment of CRT response. Patients will receive standard treatment for their malignancy. This study does not involve a treatment intervention.

\section{Study schematic}

All patients will receive standard treatment consisting of neoadjuvant CRT followed by surgery, and have MRI and PET performed at three time-points (Fig. 1); pre-CRT, during-CRT (week 3 of CRT), and post-CRT (within 1 week prior to surgery).

\section{Patient selection \\ Inclusion criteria}

1. Age greater than 18

2. Stage II or III rectal adenocarcinoma, defined as $\mathrm{T} 3$ - T4 and/or node positive disease (N1-2), without distant metastatic disease (M0)

3. No evidence of metastatic disease on computed tomography (CT) chest/abdomen/pelvis

4. Undergoing treatment regimen consisting of neoadjuvant CRT (Radiotherapy 50.4Gy in 28 fractions delivered using 3D-conformal or VMAT 


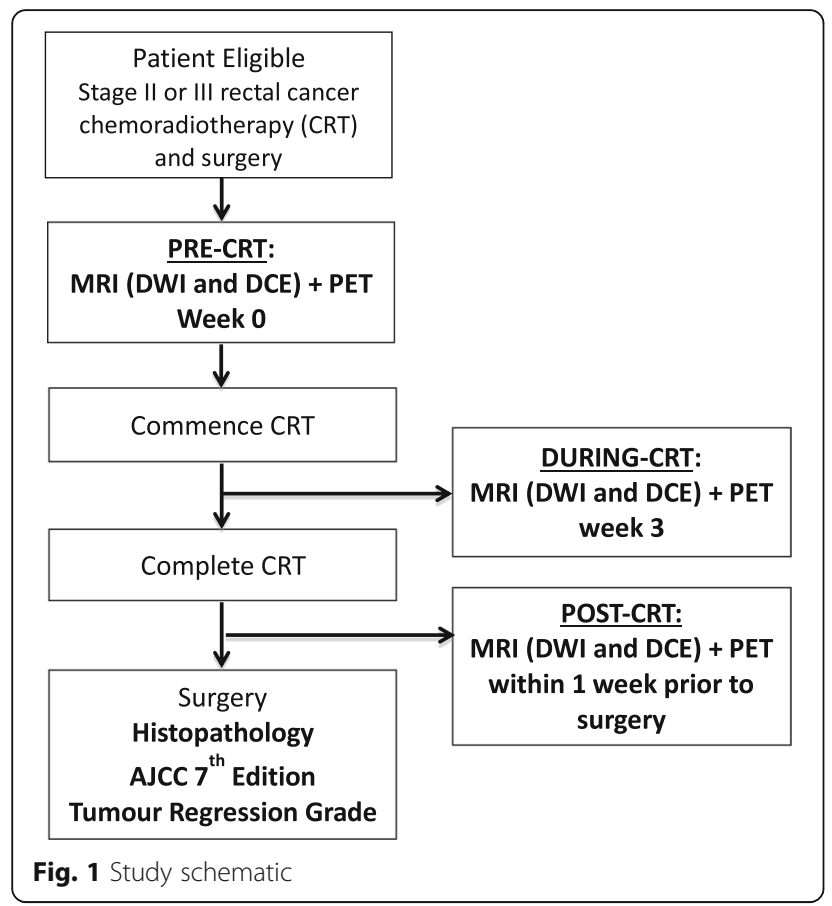

technique concurrent with infusional 5-fluorouracil or oral capecitabine) followed by primary surgery.

\section{Exclusion criteria}

1. Other malignancy

2. Active inflammatory bowel disease

3. Contraindication to MRI:

- Implanted ferromagnetic metal eg. Intraocular metal

- Pacemaker/Implantable defibrillator

- Extreme claustrophobia

\section{Treatment}

Patients are to undergo standard treatment, consisting of neo-adjuvant long course CRT (as detailed above) followed by surgery, as recommended by treating team. There will be no change to the patient's treatment from participating in this study.

\section{Imaging study procedures}

\section{Timing of multi-parametric MRI and PET}

1. Pre-CRT: week -2 to 0

2. During-CRT: Week 3 of CRT (early as possible during week 3)

3. Post-CRT: Post completion of CRT, within 1 week prior to surgery

\section{MRI technique}

All MRI scans will be acquired on the 3 Tesla Siemens Skyra (Magnetom, Erlangen, Germany) dedicated MRISimulator within the Radiation Oncology department. A 32-channel spine coil integrated in the patient table will be used in combination with an 18-channel phase array surface coil strapped firmly around the pelvis. Butylscopolamine (Buscopan) $20 \mathrm{mg}$ will be administered intravenously prior to acquisition of functional sequences (DWI and DCE) to reduce rectal motion.

\section{MRI safety screening}

All patients will undergo MRI safety screening.

Screening of suitability for gadolinium-based MRI contrast

1. Require documentation of normal renal function within three months $(\mathrm{eGFR}>=60 \mathrm{ml} / \mathrm{min} / 1.73 \mathrm{~m} 2$

2. Contra-indications to use of gadolinium-based MRI contrast/DCE:

1. Renal impairment eGFR $<60 \mathrm{ml} / \mathrm{min} / 1.73 \mathrm{~m} 2$

2. Acute kidney injury

3. Previous allergic reaction to gadolinium-based MRI contrast.

\section{MRI sequences}

T2-weighted turbo spin echo images - Acquired in 3 planes with $2 \mathrm{~mm}$ slice thickness and bandwidth $440 \mathrm{~Hz} / \mathrm{Px}$ in 1 concatenation: (i) sagittal, (ii) axial oblique, angulated perpendicular to the long tumour axis, and (iii) axial. A T2-w HASTE sequence is additionally acquired in the coronal plane.

\section{Diffusion weighted imaging (DWI)}

- Readout segmented diffusion technique (RESOLVE)

- Axial orthogonal plane with b-values 50 and $800 \mathrm{~s} / \mathrm{mm}^{2}$, and $1 \& 3$ signal averages respectively.

- ADC maps and calculated $b=1400 \mathrm{~mm} / \mathrm{s}^{2}$ images produced as part of protocol.

- In-plane resolution of $1.1 \times 1.1 \mathrm{~mm}$ and $4 \mathrm{~mm}$ slice thickness.

\section{Dynamic contrast enhanced (DCE) imaging}

- Pre-contrast T1-weighted VIBE scans with TR $4.09 \mathrm{~ms}$, TE $1.35 \mathrm{~ms}$, and flip angles $2^{\circ}$ and $15^{\circ}$ to calculate native $\mathrm{T} 1$.

- DCE acquired with time-resolved angiography with stochastic trajectories (TWIST) technique acquired over 60 phases, with a temporal resolution of $5.28 \mathrm{~s}$, bandwidth $440 \mathrm{~Hz} / \mathrm{Px}$, TR $3.67 \mathrm{~ms}$ and TE $1.48 \mathrm{~ms}$. Gadoversetamide (Optimark) $0.1 \mathrm{mM} / \mathrm{kg}$ injected after 3 phases acquired. 


\section{$\mathrm{PET} / \mathrm{CT}$ technique}

${ }^{18} \mathrm{~F}-\mathrm{FDG}$ PET/CT will be acquired on the GE Discovery710 PET-CT. Patients need to fast for at least $4 \mathrm{~h}$ prior to $4.29 \pm 0.34$ (mean $\pm \mathrm{SD}$ ) $\mathrm{MBq} / \mathrm{kg}{ }^{18} \mathrm{~F}-\mathrm{FDG}$ injection and have blood glucose levels $<10 \mathrm{mmol} / \mathrm{L}$. All PET scans will be acquired in three-dimensional mode from the mid-brain to proximal femora with an acquisition time of 1.5-2.5 min per bed position, after an ${ }^{18} \mathrm{~F}$-FDG uptake time of $60 \mathrm{~min}$. PET data will be reconstructed into a $256 \times 256$ matrix size with slice thickness of $3.3 \mathrm{~mm}$ using GE VUE Point FX (Time of Flight) algorithm. All PET/CT scans will be evaluated on the Advantage Workstation (GE Healthcare) using the AW VolumeShare 5 software and PET-VCAR (Volume Computer-Assisted Reading) protocol.

\section{Clinical assessment and acute toxicity grading}

1. Radiation Oncology clinical assessments and formal acute toxicity scoring will be performed at the following time points:

a. Pre-CRT: Baseline assessment prior to commencement of treatment

b. During-CRT: Weekly during radiotherapy treatment reviews

c. Post-CRT: At 2 weeks post-completion of CRT, and within 1 week prior to surgery (ideally at time of post-CRT MRI).

2. Acute toxicity will be scored according to the 'Common Terminology Criteria for Adverse Events (CTCAE) Version 4.0.

\section{Histopathology and tumour regression grade criteria}

Histopathological assessment will be undertaken by a dedicated pathology team, with all reporting/TRG assessment by pathologists with sub-specialisation in gastrointestinal pathology. For the study it is a requirement that the whole of the original tumour site should be embedded for microscopic assessment.

MRI will be correlated with histopathological TRG using the modified classification of Ryan et al. [15] set out in the AJCC Cancer Staging Manual, 7th Edition as follows:

TRG O (complete response) - no viable cancer cells.

TRG 1 (moderate response) - single cells or small groups of cancer cells.

TRG 2 (minimal response) - residual cancer outgrown by fibrosis.

TRG 3 (poor response) - minimal or no tumour kill; extensive residual cancer.

Patients with TRG $0-1$ will be categorized as 'good responders' and those with TRG 2-3 categorized as 'poor responders' to CRT.

\section{Multi-parametric imaging analysis}

A Radiation Oncologist with sub-specialization in gastrointestinal malignancies and a Radiologist with sub-specialization in pelvic MRI will perform all segmentations. $\mathrm{K}^{\text {trans }}$ maps will be produced by first preselecting an appropriate arterial input function, scaled by dose, based on chi-squared goodness of fit, and using a two-compartment Tofts model [16]. ADC and $\mathrm{K}^{\text {trans }}$ parameter maps will be exported in DICOM format and registered to $\mathrm{T} 2-\mathrm{w}$ axial images.

The region of interest for analysis will be defined on the entire hyper-intense primary tumour on the b-value $1400 \mathrm{~mm} / \mathrm{s} \mathrm{s}^{2}$ images. A voxel-by-voxel technique will be used to produce colour-coded maps and histograms of ADC and $K^{\text {trans }}$, and combined scatterplots for each time-point.

\section{Statistical considerations}

A target of 35 patients will be recruited; this is estimated to take 24 months. For estimated pCR rate of $25 \%, 35$ patients will provide $80 \%$ power to detect an AUC $\geq 80 \%$. Poor responders rate is estimated to be in the range of $25-45 \%$ [5]. For estimated poor responder rate of $25 \%$, 35 patients will provide $80 \%$ power to detect an AUC $\geq 80 \%$. For estimated poor responder rate of $45 \%, 35$ patients will provide $80 \%$ power to detect an AUC $\geq 76 \%$.

Univariate analysis will be performed to investigate the association between each MRI parameter and tumour regression grade (AJCC 7th edition). Each MRI parameter will be correlated with pathologic complete responders (pCR - TRG 0) using a $2 \times 2$ Table. A similar analysis will be performed for good responders (TRG0-1) vs. poor responders (TRG 2-3). Receiver operator characteristics curves will be used to obtain an optimal threshold for each MRI parameter (ADC and $\mathrm{K}^{\text {trans }}$ ).

Multivariate analysis will be performed to investigate the association between multi-parametric MRI and tumour regression grade. The multi-parametric MRI parameters that will be used for multivariate analysis are $\mathrm{ADC}$ and $\mathrm{K}^{\text {trans }}$.

\section{Discussion}

MRI offers a variety of functional parameters, with each parameter offering information on various biological aspects of tumour. Multi-parametric image analysis has become increasingly relevant in cancer therapy response predication, as analysis of multiple parameters can provide a more complete physiological assessment of tumour [17]. The rectum is a particularly challenging anatomy to image and provide robust functional datasets that can be examined in a serial manner. In order to provide reliable quantitative assessment of treatment response, it is important to have a standardized protocol that minimizes organ motion and provides robust serial image datasets. This study protocol describes standardized imaging procedures combining DWI and DCE, and a 3D voxel-wise multi- 


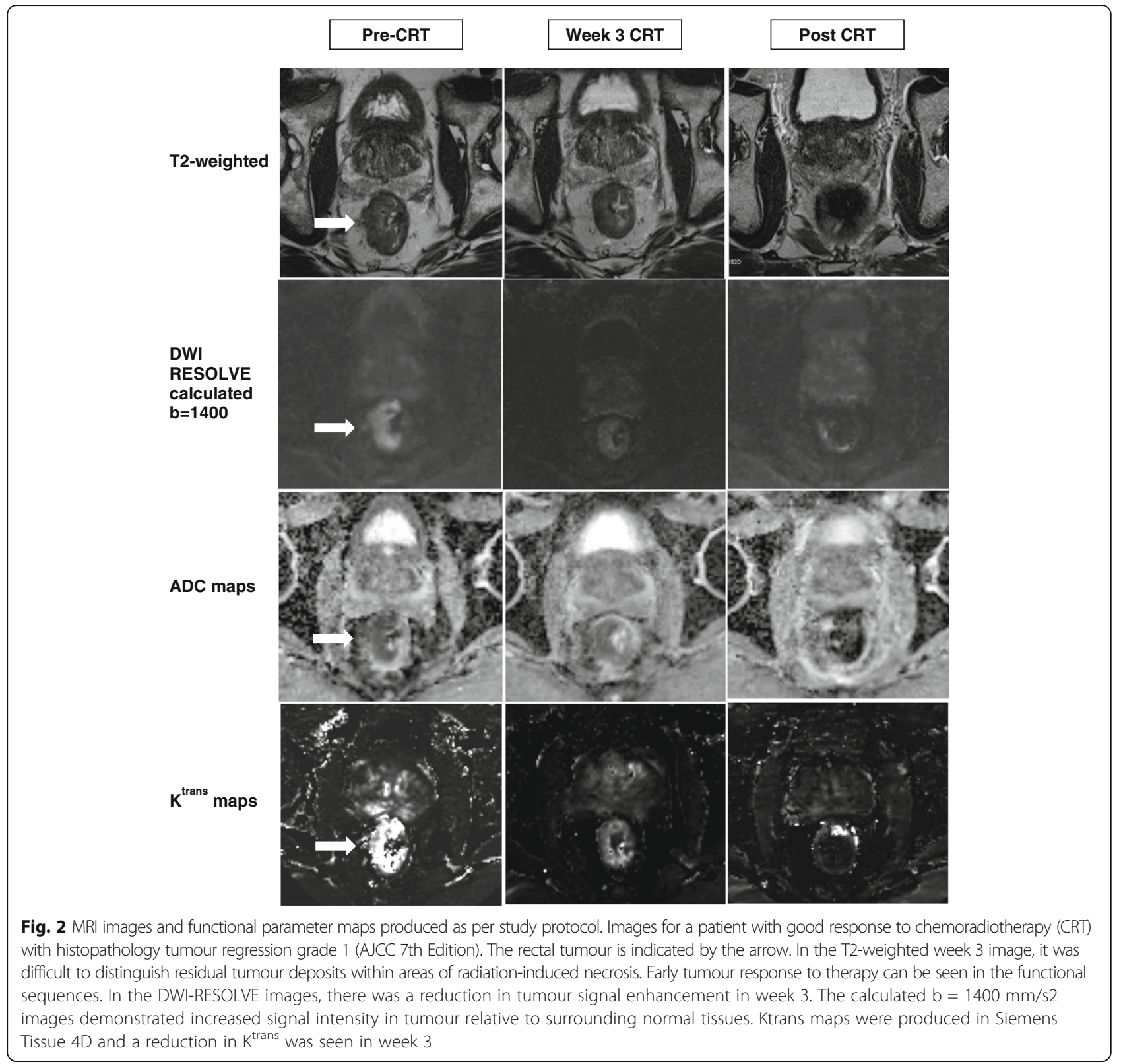

parametric analysis strategy for the assessment of tumour heterogeneity, and prediction of response to CRT in rectal cancer. Both DWI and DCE techniques in this protocol have improvements compared to previously used techniques. The DWI RESOLVE sequence used in this study protocol has previously been shown to be more robust with respect to geometrical distortions, compared to DWI standard echo planar images [18]. The calculated $\mathrm{b}=1400 \mathrm{~mm} / \mathrm{s}^{2}$ images gains from both extra sensitivity and reduced noise of a calculated high b-value. The short temporal resolution of $5.28 \mathrm{~s}$ in DCE-TWIST will enable adequate sampling of the rapid wash-in of contrast into tumour. For acquisition of DCE images, the administration of butylscopolamine is crucial in eliminating rectal motion and ensuring accurate signal intensity for each pixel over the 60 time-points. Figure 2 shows examples of the good quality functional images with minimal distortion and corresponding parameter maps that are able to be acquired with this study protocol.

If validated, multi-parametric MRI combining DWI and DCE can be used to stratify management in rectal cancer patients. Accurate imaging prediction of patients with a complete response to CRT would enable a 'wait and watch' approach, avoiding surgical morbidity in these patients. Consistent and reliable quantitation from standardized protocols is essential in order to establish optimal thresholds of $\mathrm{ADC}$ and $\mathrm{K}^{\text {trans }}$ and permit the role of multi-parametric MRI for early treatment prediction to be properly evaluated. 


\section{Abbreviations}

ADC: Apparent diffusion co-efficient; CRT: Chemoradiotherapy; CT: Computed tomography; DCE: Dynamic contrast enhanced; DWl: Diffusion weighted imaging; LARC: Locally advanced rectal cancer; MRI: Magnetic resonance imaging; pCR: Pathologic complete response; RESOLVE: Read-out segmented sequence; ROI: Region of interest; T2-W: T2-weighted; TRG: Tumour regression grade; TWIST: Time-resolved angiography with stochastic trajectories

\section{Acknowledgments}

Not applicable.

\section{Funding}

This study has been funded by the Royal Australian and New Zealand College of Radiologists (RANZCR) with a Withers and Peters $\$ 25,000$ competitive research grant. RANZCR was not involved in the design of this study protocol and will not be involved in data collection or analysis.

\section{Availability of data and materials}

Not applicable.

\section{Authors' contributions}

All authors participated in the design and write-up of this study protocol. All authors contributed to, read and approved the final manuscript. TP is the principal investigator of the study, participated in all components of the study design and wrote the study protocol. TP, KW, MLee and MBB conceived the study idea, and contributed to the study design and protocol. TP, GL, RR, $\mathrm{DM}$, and MLin participated in the design of the imaging protocol. $\mathrm{CH}$ and JSS participated in the design of the histopathology protocol.

\section{Ethics approval and consent to participate}

This study and associated patient information sheets have been approved by the South Western Sydney Local Health District Human Research and Ethics Committee (HREC). Reference HREC/13/LPOOL/158, local project number 13/ 097a, 13/097b, 13/097c, Sub-study Rectal Cancer. All patients provide written informed consent prior to any study-related procedures.

\section{Consent for publication}

All images have been de-identified. Written informed consent was obtained from all patients participating in this study for the publication of de-identified images and data.

\section{Competing interests}

The authors declare that they have no competing interests.

\section{Author details}

Department of Radiation Oncology, Liverpool Cancer Therapy Centre, Liverpool Hospital, South Western Sydney Local Health District, Sydney, Australia. ${ }^{2}$ Sydney West Radiation Oncology Network, Westmead, Blacktown and Nepean Hospitals, Sydney, Australia. ${ }^{3}$ Faculty of Medicine, University of New South Wales, Sydney, Australia. ${ }^{4}$ Ingham Institute for Applied Medical Research, Sydney, Australia. ${ }^{5}$ Department of Radiology, Prince of Wales Hospital, Sydney, Australia. ${ }^{6}$ Department of Anatomical Pathology, Liverpoo Hospital, Sydney, Australia. ${ }^{7}$ School of Medicine, Western Sydney University, Sydney, Australia. ${ }^{8}$ Department of Nuclear Medicine, Liverpool Hospital, Sydney, Australia. ${ }^{9}$ Faculty of Radiation and Medical Physics, University of Wollongong, Sydney, Australia.

Received: 22 January 2017 Accepted: 26 June 2017

Published online: 04 July 2017

\section{References}

1. Sauer R, Liersch T, Merkel S, Fietkau R, Hohenberger W, Hess C, Becker H, Raab HR, Villanueva MT, Witzigmann $H$, et al. Preoperative versus postoperative chemoradiotherapy for locally advanced rectal cancer: results of the German CAO/ARO/AIO-94 randomized phase III trial after a median follow-up of 11 years. J Clin Oncol. 2012;30(16):1926-33.

2. National Comprehensive Cancer Network (NNCN): NCCN Clinical Practice Guidelines in Oncology Rectal Cancer Version 3.2015. 2015.

3. National Institute for Health and Care Excellence (NICE): Colorectal cancer the diagnosis and management of colorectal cancer. NICE clinical guideline 1312014
4. Maas M, Nelemans PJ, Valentini V, Das P, Rödel C, Kuo L-J, Calvo FA, GarcíaAguilar J, Glynne-Jones R, Haustermans K, et al. Long-term outcome in patients with a pathological complete response after chemoradiation for rectal cancer: a pooled analysis of individual patient data. Lancet Oncol. 2010;11(9):835-44

5. Valentini V, Coco C, Cellini N, Picciocchi A, Fares MC, Rosetto ME, Mantini G, Morganti AG, Barbaro B, Cogliandolo S, et al. Ten years of preoperative chemoradiation for extraperitoneal T3 rectal cancer: acute toxicity, tumor response, and sphincter preservation in three consecutive studies. Int J Radiat Oncol Biol Phys. 2001;51(2):371-83.

6. McCarthy K, Pearson K, Fulton $\mathrm{R}$, Hewitt J. Pre-operative chemoradiation for non-metastatic locally advanced rectal cancer. The Cochrane database of systematic reviews. 2012;12:CD008368.

7. Renehan AG, Malcomson L, Emsley R, Gollins S, Maw A, Myint AS, Rooney PS, Susnerwala S, Blower A, Saunders MP, et al. Watch-and-wait approach versus surgical resection after chemoradiotherapy for patients with rectal cancer (the OnCoRe project): a propensity-score matched cohort analysis. Lancet Oncol.

8. Maas M, Beets-Tan RG, Lambregts DM, Lammering G, Nelemans PJ, Engelen SM, van Dam RM, Jansen RL, Sosef M, Leijtens JW, et al. Wait-and-see policy for clinical complete responders after chemoradiation for rectal cancer. J Clin Oncol. 2011:29(35):4633-40.

9. Habr-Gama A, Perez RO, São Julião GP, Proscurshim I, Gama-Rodrigues J. Nonoperative approaches to rectal cancer: a critical evaluation. Semin Radiat Oncol. 2011;21(3):234-9.

10. van der Paardt MP, Zagers MB, Beets-Tan RGH, Stoker J, Bipat S. Patients who undergo preoperative Chemoradiotherapy for locally advanced recta cancer restaged by using diagnostic MR imaging: a systematic review and meta-analysis. Radiology. 2013;269(1):101-12.

11. Joye I, Deroose CM, Vandecaveye V, Haustermans K. The role of diffusionweighted MRI and (18)F-FDG PET/CT in the prediction of pathologic complete response after radiochemotherapy for rectal cancer: a systematic review. Radiother Oncol. 2014;113(2):158-65.

12. Intven M, Reerink O, Philippens ME. Dynamic contrast enhanced MR imaging for rectal cancer response assessment after neo-adjuvant chemoradiation. J Magn Reson Imaging. 2015;41(6):1646-53.

13. George M, Dzik-Jurasz A, Padhani A, Brown G, Tait D, Eccles S, Swift R. Noninvasive methods of assessing angiogenesis and their value in predicting response to treatment in colorectal cancer. Br J Surg. 2001;88(12):1628-36.

14. Lim JS, Kim D, Baek SE, Myoung S, Choi J, Shin SJ, Kim MJ, Kim NK, Suh J, Kim KW, et al. Perfusion MRI for the prediction of treatment response after preoperative chemoradiotherapy in locally advanced rectal cancer. Eur Radiol. 2012;22(8):1693-700.

15. Ryan R, Gibbons D, Hyland J. Pathological response following long course neoadjuvant chemoradiotherapy for locally advanced rectal cancer. Histopathology. 2005:47:141-6.

16. Tofts PS, Brix G, Buckley DL, Evelhoch JL, Henderson E, Knopp MV, Larsson HB, Lee TY, Mayr NA, Parker GJ, et al. Estimating kinetic parameters from dynamic contrast-enhanced T(1)-weighted MRI of a diffusable tracer: standardized quantities and symbols. J Magn Reson Imaging. 1999;10(3): 223-32.

17. Yankeelov T, Pickens D, Price R (eds.): Quantitative MRI in Cancer (Imaging in Medical Diagnosis and Therapy). Boca Raton, FL 33487-2742: CRC Press Taylor \& Francis Group; 2012.

18. Liney GP, Holloway L, Harthi TMA, Sidhom M, Moses D, Juresic E, Rai R, Manton DJ. Quantitative evaluation of diffusion-weighted imaging techniques for the purposes of radiotherapy planning in the prostate. $\mathrm{Br}$ J Radiol. 2015;88(1049):20150034. 